OPEN ACCESS

Edited by:

Colin H. Macphee,

GlaxoSmithKline (United States),

United States

Reviewed by:

Milica S. Prostran,

University of Belgrade, Serbia

Claudio de Lucia,

Temple University, United States

*Correspondence:

$\mathrm{HaO} \mathrm{Li}$

lihao19880810@hotmail.com

tThese authors have contributed equally to this work as first authors.

Specialty section:

This article was submitted to Cardiovascular and Smooth Muscle

Pharmacology,

a section of the journal

Frontiers in Pharmacology

Received: 09 March 2018 Accepted: 25 April 2018

Published: 15 May 2018

Citation:

Gu Z-C, Ma X-W, Zheng X-Y, Shen L,

Shi F-H and Li H (2018) Left Atrial Appendage Thrombus Formation in a

Patient on Dabigatran Therapy Associated With ABCB1 and CES-1

Genetic Defect.

Front. Pharmacol. 9:491.

doi: 10.3389/fphar.2018.00491

\section{Left Atrial Appendage Thrombus Formation in a Patient on Dabigatran Therapy Associated With ABCB1 and CES-1 Genetic Defect}

\author{
Zhi-Chun Gu ${ }^{1+}$, Xiao-Wei Ma ${ }^{2+}$, Xiao-Yuan Zheng ${ }^{3+}$, Long Shen ${ }^{4}$, Fang-Hong Shi ${ }^{1}$ and \\ Hao Li ${ }^{5 *}$ \\ ${ }^{1}$ Department of Pharmacy, Renji Hospital, School of Medicine, Shanghai Jiaotong University, Shanghai, China, ${ }^{2}$ Department \\ of Clinical Laboratory, Renji Hospital, School of Medicine, Shanghai Jiaotong University, Shanghai, China, ${ }^{3}$ Department of \\ Pharmacy, Affiliated Hospital of Jiangnan University, Wuxi, China, ${ }^{4}$ Department of Cardiology, Renji Hospital, School of \\ Medicine, Shanghai Jiaotong University, Shanghai, China, ${ }^{5}$ Department of Pharmacy, Shanghai Children's Medical Center, \\ School of Medicine, Shanghai Jiaotong University, Shanghai, China
}

Dabigatran, directly targeting thrombin, is widely used for the prevention of stroke in nonvalvular atrial fibrillation (NVAF). We reported a rare case of left atrial appendage thrombus formation in a persistent NVAF patient despite the 31 months uninterrupted treatment with dabigatran $110 \mathrm{mg}$ twice daily. The patient is a carrier of ABCB1 variant alleles with 7 heterozygote single nucleotide polymorphisms (SNPs: rs4148738, rs2235046, rs1128503, rs10276036, rs1202169, rs1202168, rs1202167) as well as CES-1 variant alleles with 2 homozygote SNPs (rs2244613 and rs4122238) and 2 heterozygote SNPs (rs8192935 and rs4580160), which may contribute to the changes of dabigatran plasma concentration. In addition, Drug-drug interaction with atorvastatin may also play a role to decrease dabigatran plasma concentration. There are only four such cases till date, of which had thrombus in the left atrium, reported in the literature. We firstly reported the documented case in a Chinese patient carrying multiple alleles of ABCB1 and CES-1, who suffered from thrombus in the left atrial appendage despite long-term anticoagulation with dabigatran. More clinical data are required to elucidate the impact of CES-1 and ABCB1 polymorphism on dabigatran pharmacokinetics, especially for Asian.

Keywords: dabigatran, left atrial appendage thrombus, genetic polymorphism, ABCB1, CES-1, drug-drug interaction

\section{INTRODUCTION}

Warfarin, one of the vitamin K-dependent antagonists (VKAs), is the most commonly used oral anticoagulant (Mega and Simon, 2015). Although warfarin has been used clinically for more than 60 years, several challenges including bleeding complications have been noted, which are one of the primacy causes of severe adverse drug events (Crowther et al., 2000; Wysowski et al., 2007). The inherent limitations of warfarin, comprising narrow therapeutic window, intra-patient variability, and numerous food-drug interaction and drug-drug interaction, lead to a need for more elaborative anticoagulation monitoring (Johnson et al., 2011; Pirmohamed et al., 2013). Different from warfarin, non-VKA oral anticoagulants (NOACs), which directly target thrombin 
or Xa factor, have been approved for the prevention of stroke and systemic embolism in non-valvular atrial fibrillation without necessary of routine blood monitoring (López-López et al., 2017). Dabigatran etexilate, the prodrug of dabigatran, has the lowest bioavailability of present NOACs of 3 7\% (Mega and Simon, 2015), which is rapidly converted by esterases-1 (CES-1) to dabigatran after administration (Stangier and Clemens, 2009; Laizure et al., 2014). In addation, dabigatran etexilate is a substrate of the P-glycoprotein intestinal efflux transporter $(P$ $g p$ ), which also known as ATP-binding cassette sub-family B member 1 (ABCB1) (Paré et al., 2013). P-gp is one of the drug transporters expressed in gastrointestinal tract which involves in the efflux of various kinds of drugs into lumen (Ambudkar et al., 2003). Thus, strong P-gp inhibitors can increase dabigatran bioavailability by 12-23\% (Liesenfeld et al., 2011; Paré et al., 2013; Kishimoto et al., 2014). Unlike Xa factor inhibitors, oxidoreductases or cytochrome P450 enzymes are not involved in the metabolism of dabigatran (Blech et al., 2008). As the renal clearance of dabigatran is $80 \%$, renal function should be monitored regularly in patients taking dabigatran (Mega and Simon, 2015). Accordingly, changes in the process of absorption or elimination could have a great effect on dabigatran plasma concentrations (Antonijevic et al., 2017; Favaloro et al., 2017). We reported a dabigatran-treated patient who presented with severe left atrial appendage thrombus formation, of whom $A B C B 1$ and CES1 genetic polymorphism and drug-drug interaction may have been the contributing factors. The patient gave his written informed consent for publication of this report.

\section{CASE PRESENTATION}

The present patient is a 70-year-old Chinese male with paroxysmal atrial fibrillation, complicating hypertension, type 2 diabetes mellitus, coronary heart disease, cerebral infarction, and lost binocular vision. The patient had received dabigatran etexilate $110 \mathrm{mg}$ twice daily since April 30th, 2015 for the prevention of stroke and systemic embolism. Before the introduction of dabigatran, several drugs, including atorvastatin, have been taking for years (Table 1). The patient was admitted to the department of cardiology on November 9th 2017 due to the recurrence of cardio-embolic stroke. Vital signs were normal with blood pressure of $133 / 73 \mathrm{mmHg}$ and heart rate of 73 rates per minute. Transesophageal echocardiography on admission showed a large thrombus in the left atrial appendage (Figure 1, Video 1), the enlarged left atrial diameter of $46 \mathrm{~mm}$ (reference: $30-40 \mathrm{~mm}$ ) as well as depressed left ventricular function with left ventricular ejection fraction of 52\% (reference: 55\%) (Table 2). Laboratory data on admission were within normal limits expect for serum creatinine of $115 \mathrm{umol} / \mathrm{L}$ (reference: 45-104 umol/L) and $\mathrm{B}$ type natriuretic peptide (BNP) of $188 \mathrm{pg} / \mathrm{mL}$ (reference: $0.0 \sim 100 \mathrm{pg} / \mathrm{mL}$ ) (Table 2). Dabigatran was stopped at admission, and enoxaparin was administrated with the combination of warfarin since November 8th, 2017 for 20 days till November 28th, 2017. Finally, the patient was treated with coronary artery bypass grafting (CABG), atrial fibrillation ablation, left atrial appendage excision and pericardial drainage plus cardiac
TABLE 1 | Drugs administration during 3 years

\begin{tabular}{|c|c|c|c|c|}
\hline Starting Time & $\begin{array}{l}\text { June 21st, } \\
2014\end{array}$ & $\begin{array}{l}\text { Nov. 30th, } \\
2014\end{array}$ & $\begin{array}{l}\text { Apr. 4th, } \\
2015\end{array}$ & $\begin{array}{l}\text { Nov. 11th, } \\
2017\end{array}$ \\
\hline Stop Time & $\begin{array}{l}\text { Nov. 29th, } \\
2014\end{array}$ & $\begin{array}{l}\text { Apr. 3rd, } \\
2015\end{array}$ & $\begin{array}{l}\text { Nov. 8th, } \\
2017\end{array}$ & $\begin{array}{l}\text { Dec. 20th, } \\
2017\end{array}$ \\
\hline $\begin{array}{l}\text { Fosinopril Sodium } \\
\text { Tablets }\end{array}$ & NK & - & - & - \\
\hline $\begin{array}{l}\text { Amlodipine Besylate } \\
\text { Tablets }\end{array}$ & $5 \mathrm{mg}$ bid & $5 \mathrm{mg}$ bid & $5 \mathrm{mg}$ bid & - \\
\hline $\begin{array}{l}\text { Metoprolol Tartrate } \\
\text { Tablets }\end{array}$ & NK & $25 \mathrm{mg}$ bid & $25 \mathrm{mg}$ bid & - \\
\hline $\begin{array}{l}\text { Clopidogrel Hydrogen } \\
\text { Sulphate Tablets }\end{array}$ & - & $75 \mathrm{mg} \mathrm{qd}$ & - & - \\
\hline Atorvastatin Tablets & - & $20 \mathrm{mg}$ qd & $20 \mathrm{mg}$ qd & - \\
\hline $\begin{array}{l}\text { Metformin } \\
\text { Hydrochloride Tablets }\end{array}$ & - & $500 \mathrm{mg}$ qd & $500 \mathrm{mg}$ qd & - \\
\hline $\begin{array}{l}\text { Dabigatran Etexilate } \\
\text { Capsules }\end{array}$ & - & - & $110 \mathrm{mg}$ bid & - \\
\hline $\begin{array}{l}\text { Clonidine } \\
\text { Hydrochloride Tablets }\end{array}$ & - & - & $75 \mu \mathrm{g} \mathrm{qd}$ & - \\
\hline Olmesartan Medoxomil & - & - & $20 \mathrm{mg} \mathrm{qd}$ & - \\
\hline Warfarin Tablets & - & - & - & $2.5 \mathrm{mg}$ qn \\
\hline
\end{tabular}

NK, not known

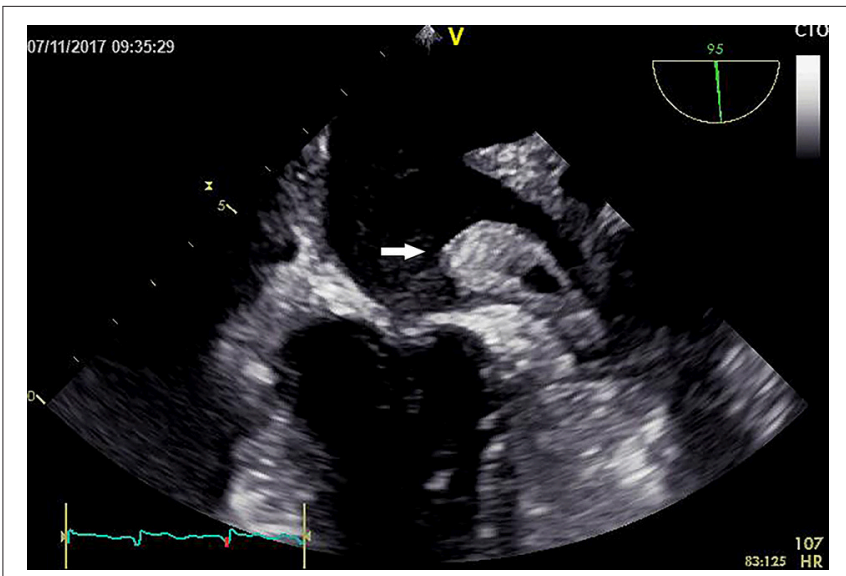

FIGURE 1 | Transesophageal echocardiogram showed the large left atrial thrombus.

temporary pacemaker implantation in November 29th, 2017. He was discharged day 15 post-operation on treatment with warfarin $2.5 \mathrm{mg}$ daily. At 3 months follow up, the patient has been doing well without any evidence of recurrent thrombotic events.

\section{INVESTIGATIONS}

Clinical investigations were performed to assess the causes of potential decreased dabigatran effects at therapeutic doses. They included patient's characteristic, co-administrated drugs, and genotyping of $A B C B 1$ and $C E S 1$. 
TABLE 2 | Results of main laboratory test.

\begin{tabular}{|c|c|c|c|c|c|c|c|c|c|c|}
\hline Index (normal range) & Nov. 8th & 10th & 12th & 14th & 16th & 20th & 23th & 27th & 29th & 30th \\
\hline \multicolumn{11}{|l|}{ REGULAR BLOOD ANALYSIS } \\
\hline White blood cell count (3.97 9.15 × 10^9/L) & 8.53 & - & - & - & - & - & - & - & 15.65 & 24.86 \\
\hline Neutrophils\% (50 70\%) & 54.8 & - & - & - & - & - & - & - & 85.0 & 89.9 \\
\hline Platelet count $\left(85 \sim 303 \times 10^{\wedge}\right.$ g/L) & 200 & - & - & - & - & - & - & - & 133 & 121 \\
\hline Red blood cell count $\left(4.09 \sim 5.74 \times 10^{\wedge} 12 / L\right)$ & 5.56 & - & - & - & - & - & - & - & 3.85 & 3.61 \\
\hline Triglyceride (<1.7 mmol/L) & 2.75 & - & - & - & - & - & - & - & - & - \\
\hline Total cholesterol (< 5.72 mmol/L) & 3.38 & - & - & - & - & - & - & - & - & - \\
\hline Total bilirubin (3.4 17.1 umol/L) & 13.5 & - & - & - & - & - & - & - & 36.0 & - \\
\hline High density lipoprotein (0.9 2.0 mmol/L) & 0.61 & - & - & - & - & - & - & - & - & - \\
\hline Low density lipoprotein (0.9 2.0 mmol/L) & 1.70 & - & - & - & - & - & - & - & - & - \\
\hline Non high density lipoprotein (1.8 4.1 mmol/L) & 2.77 & - & - & - & - & - & - & - & - & - \\
\hline Creatinine (45 104 umol/L) & 115.0 & - & 119.0 & - & 144.0 & 144.0 & 150.0 & - & 138.0 & 157.0 \\
\hline Blood ketone body (negative) & Negative & - & - & - & - & - & - & - & - & - \\
\hline Blood ammonia (9 30 umol/L) & 72.48 & - & - & - & - & - & - & - & - & - \\
\hline Erythrocyte sedimentation rate $(0.0 \sim 15.0 \mathrm{~mm} / \mathrm{h})$ & 29 & - & - & - & - & - & - & - & - & - \\
\hline Glycosylated hemoglobin Hba1C (4 6\%) & 7.0 & - & - & - & - & - & - & - & - & - \\
\hline $2 \mathrm{~h}$ blood glucose (<7.8 mmol/l) & 8.32 & - & - & - & - & - & - & - & - & - \\
\hline High sensitive $C$ reactive protein $(0 \sim 3 \mathrm{mg} / \mathrm{L})$ & 2.9 & - & - & - & - & - & - & - & - & - \\
\hline B type natriuretic peptide $(0.0 \sim 100$ pg/mL) & 188.00 & 160.0 & - & - & - & - & - & - & - & - \\
\hline \multicolumn{11}{|l|}{ MYOCARDIAL INFARCTION MARKERS } \\
\hline Troponin (<0.04 ng/mL) & 0.01 & - & - & - & - & - & - & - & 10.65 & 4.01 \\
\hline Creatine kinase $(0.6 \sim 6.3 \mathrm{ng} / \mathrm{mL})$ & 0.7 & - & - & - & - & - & - & - & 639 & - \\
\hline Myoglobin (17.4 105.7 ng/mL) & 33.10 & - & - & - & - & - & - & - & - & - \\
\hline \multicolumn{11}{|l|}{ BLOOD COAGULATION ANALYSIS } \\
\hline Fibrin degradation product $(0 \sim 5 \mu \mathrm{g} / \mathrm{ml})$ & 5.90 & 10.10 & - & - & - & - & - & - & - & - \\
\hline Thrombin time (14 21s) & 20.50 & 20.70 & - & - & - & - & - & - & - & - \\
\hline Prothrombin time $(9.4 \sim 12.5 \mathrm{~s})$ & 12.00 & 14.70 & - & 13.5 & 18.50 & 36.00 & 37.30 & 11.4 & - & - \\
\hline Fibrinogen (2.00 4.00 g/L) & 3.75 & 3.67 & - & - & - & - & - & - & - & - \\
\hline Activated partial prothrombin time (25 33.8s) & 28.20 & 30.10 & - & - & - & - & - & - & - & - \\
\hline Prothrombin INR (0.8 1.15) & 1.01 & 1.23 & - & 1.13 & 1.65 & 2.99 & 3.10 & 1.03 & - & - \\
\hline D-Dimer (0 0.5 $\mu \mathrm{g} / \mathrm{mL})$ & 0.95 & 1.48 & - & - & - & - & - & - & - & - \\
\hline \multicolumn{11}{|l|}{ ECHOCARDIOGRAPHY } \\
\hline Internal diameter of the aortic root (28 40 mm) & 37 & - & - & - & - & - & - & - & - & - \\
\hline Left atrial diameter (30 40 mm) & 46 & - & - & - & - & - & - & - & - & - \\
\hline The left ventricular ejection fraction (55\%) & 52 & - & - & - & - & - & - & - & - & - \\
\hline Left ventricular end diastolic diameter (38 52 mm) & 48 & - & - & - & - & - & - & - & - & - \\
\hline
\end{tabular}

Data were collected from Nov. 8th 2017 to Nov. 30th 2017. Nov., November; -, No tested.

\section{$A B C B 1$ and CES1 Genotyping}

Genomic DNA was extracted from whole blood $(200 \mu \mathrm{l})$ using the QIAamp DNA blood mini kit (QIAGEN, Hombrechtikon, Switzerland). ABCB1 (rs4148738, rs2235046, rs1128503, rs10276036, rs1202169, rs1202168, and rs1202167) and CES1 (rs8192935, rs2244613, and rs4122238) and CES1P2 (rs4580160 and rs4784563) polymorphisms were determined by Sanger sequencing. The polymerase chain reaction primers were presented in Table 3. Sequencing was performed on $1 \mu l$ of the purified mixture using the BigDye Terminator v1.1 Cycle Sequencing kit (Life Technologies, Warsaw, Poland) and an ABI 3130 Automatic Capillary DNA Sequencer.

\section{RESULTS}

Several reasons that contributed to treatment failure with dabigatran should be considered in this case: (1) the patient is a 70-year-old Chinese male and had a moderate renal insufficiency (estimated glomerular filtration rate of $55 \mathrm{~mL} / \mathrm{min}$ ); (2) the drug-drug interaction between dabigatran and atorvastatin was present; (3) as shown in Table 4, The patient is a heterozygote carrier of $A B C B 1$ variant alleles with 7 heterozygote single nucleotide polymorphisms (SNPs: rs4148738, rs2235046, rs1128503, rs10276036, rs1202169, rs1202168, rs1202167) as well as CES-1 variant alleles with 2 homozygote SNPs (rs2244613 and rs4122238) and 2 heterozygote SNPs (rs8192935 and rs4580160). 


\section{DISCUSSION}

We described the case of an old dabigatran-treated patient who presented with left atrial appendage thrombus formation despite the 31 months uninterrupted dabigatran therapy. Laboratory investigations showed a serum creatinine of $115 \mu \mathrm{mol} / \mathrm{L}$ and estimated glomerular filtration rate of $55 \mathrm{~mL} / \mathrm{min}$, which indicated a moderate renal insufficiency. Given that more than $80 \%$ of dabigatran is eliminated via urine, renal insufficiency was probably a contributing factor to increase the susceptibility of dabigatran. Furthermore, dabigatran may not suitable for elderly person due to insufficient renal function and correspondingly increased risk of bleeding. However, the mutation of both $A B C B 1$ and $C E S-1$ alleles may lead to a significant decrease of dabigatran blood concentration. The $A B C B 1$ gene encodes for $P-g p$, and $P$ $g p$ is an ATP-dependent drug efflux pump (Verhalen et al., 2017). Dabigatran etexilate, but not dabigatran, is a $P$ - $g p$ substrate. $P-g p$ inhibitors increase dabigatran bioavailability by $10-20 \%$

TABLE 3 | The polymerase chain reaction primers of ABCB1 and CES1.

\begin{tabular}{lll}
\hline SNP & Forward & Reverse \\
\hline rs4148738 & TGCTGTTGTGAGGCCC & $\begin{array}{l}\text { TाTGGTACATTAAAGAATT } \\
\text { GCCATC }\end{array}$ \\
rs2235046 & AATTAGAAAATGCAGCTG & TCTTGTCAGGTCTGAGTACC \\
rs1128503 & GAGTTCTGATGTITCTTG & GACCCTGCGGTGATCAGCAG \\
rs10276036 & TTGTGGAGAGCTGGATAAAGTG & AGCCCAGGAGGTAGAGGTTATG \\
rs1202169 & AGTGGTCTCTाTGGAAAGG & GTAGAAATTCTACCCTGC \\
rs1202168 & AGTGGTCTCTाTGGAAAGG & GTAGAACTTCTACCCTGC \\
rs1202167 & TCTGTCACCCAGGCTGGAGT & TGGTGGGTCTTACCTGATGC \\
rs8192935 & TTATATTATTAAAACATC & CATTGTCTCCTCAGGAAT \\
rs4580160 & CCATGCTAAGTATGTAGGGG & CATCCTTCTGAGATTTCTG \\
rs4784563 & ACTGCCACAGCTTCTCCAC & TCACCTACCTCCCAGCATA \\
rs2244613 & ATCAGCCTTGAGGCCTGAC & CTGCTAAAAAAAAAAAAGT \\
rs4122238 & GAGTGGAGGCGTGGTGGGAG & CCTTCACCCACAACATGCCC \\
\hline
\end{tabular}

SNP, single nucleotide polymorphism.
(Stangier and Clemens, 2009). As compared to the homozygotes for ABCB1 SNP rs4148738 (G allele), patients carrying 1 or $2 \mathrm{~A}$ alleles showed 5\% lower trough concentration (Dimatteo et al., 2016). CES-1 gene, which encodes for the liver carboxylesterase 1 enzyme, is responsible for the biotransformation of dabigatran etexilate into the active metabolite, namely, dabigatran (Wadkins et al., 2001; Redinbo et al., 2003). Paré et al. (2013) conducted a genome-wide association study, and found that the association of $A B C B 1$ and CES-1 SNPs was consistent with its effect on dabigatran blood concentration. Of which, CES-1 SNP rs2244613 is associated at genome-wide significance with trough concentration, while rs8192935 and rs4148738 are associated with peak concentration modestly (Paré et al., 2013; Ross and Paré, 2013). The minor allele of the CES-1 SNP rs8192935 is associated with a $12 \%$ decrease in adjusted peak concentration (Paré et al., 2013). The CES-1 SNP rs2244613 is associated with a $15 \%$ decrease in adjusted trough concentration per minor allele (Paré et al., 2013). Thus, the subject carrying 2 minor alleles of rs2244613 are expected to have $28 \%$ lower concentration than no-carriers (Paré et al., 2013). In agreement with genomewide association study, Gouin-Thibault I et al. (Gouin-Thibault et al., 2016) indicated that the heterozygous and homozygous of rs2244613 (CES-1) mutated groups had 14 and 26\% lower $A U C$ values as well as 13 and $43 \%$ lower $C_{\max }$ values than the wild-type groups. Of note, the frequency of the $\mathrm{T}$ allele of rs2244613 (CES-1) is 33\% in overall populations, but affects up to over $60 \%$ in Asians. Also, the frequency of the A allele of rs8192935 affects up to $76 \%$ in Asians while that is $42 \%$ in overall populations (Table 3). Therefore, the impacts on dabigatran concentrations related to rs2244613 and rs8192935 may be greater than previously postulated especially in Asians. Regretfully, no studies that focused on the relationship between CES-1 SNP and ischemic events have been carried out. The present case indicated the presence of $A B C B 1$ heterozygotes in all tested allele. Meanwhile, there is a homozygous mutation of CES1 rs2244613 and rs4122238 as well as heterozygote mutation of CES-1 rs8192935 and rs4580160. Presumably, patient carrying all these alleles may bring about the relatively low dabigatran blood

TABLE 4 | The results of ABCB1 and CES1 Genotyping.

\begin{tabular}{|c|c|c|c|c|c|c|c|}
\hline SNP & Chromosome & Position, bp & Locus & Function & Results & MAF(allel) & MAF in china \\
\hline rs4148738 & 7 & 87000985 & ABCB1 & Intron & GA & $0.38(\mathrm{~A})$ & 0.41 \\
\hline rs2235046 & 7 & 87012002 & ABCB1 & Intron & $A G$ & $0.44(G)$ & 0.69 \\
\hline rs1128503 & 7 & 87017537 & ABCB1 & Synonymous & TC & $0.42(C)$ & 0.69 \\
\hline rs10276036 & 7 & 87018134 & ABCB1 & Intron & CT & $0.43(\mathrm{~T})$ & 0.69 \\
\hline rs1202169 & 7 & 87033786 & ABCB1 & Intron & $A G$ & $0.43(G)$ & 0.69 \\
\hline rs8192935 & 16 & 54419295 & CES1 & Intron & $A G$ & $0.42(G)$ & 0.76 \\
\hline rs4580160 & 16 & 54326141 & CES1P2 & Intron & TC & $0.50(\mathrm{C})$ & 0.42 \\
\hline rs4784563 & 16 & 54333986 & CES1P2 & Intron & GG & $0.42(\mathrm{~A})$ & 0.24 \\
\hline rs2244613 & 16 & 54402110 & CES1 & Intron & $\pi$ & $0.33(\mathrm{~T})$ & 0.62 \\
\hline rs4122238 & 16 & 54414218 & CES1 & Intron & GG & $0.27(G)$ & 0.52 \\
\hline
\end{tabular}

SNP, single nucleotide polymorphism; MAF, minor allele frequency. 
concentration, subsequently leading to thrombus in the left atrial appendage. In addition, the presence of drug interaction with atorvastatin was probably another contributing factor to increase the risk of thrombosis. Atorvastatin is known as a moderate inhibitors of P-gp, which leads to an approximate 20\% decrease in dabigatran concentrations (Wessler et al., 2013; Stöllberger and Finsterer, 2015). According to a large retrospective cohort study of 91,330 Taiwanese patients with non-valvular atrial fibrillation who were treated with dabigatran (49.65\% of subjects) or another NOACs, concurrent use of atorvastatin was associated with a $29 \%$ decrease in the incidence rate ratio of major bleeding (Chang et al., 2017).

At present, there are limited data on the thrombosis in atrial fibrillation with the management of dabigatran. Luis et al. (2013) firstly reported the images which demonstrated the documented case of thrombosis in atrial fibrillation with coexistent valvular heart disease after 4 months anticoagulation with dabigatran. Sharma et al. (2014) report 2 cases of development of large left atrial thrombus in spite of the continuous treatment with dabigatran. Shah et al. (2015) reported another patient with thrombus formation in the left atrium. In all four cases, the formation of thrombus were in the left atrium but not in the left atrial appendage (Shah et al., 2015). Several proposed mechanisms might explain the reason of left atrial thrombus formation on dabigatran therapy. Firstly, dabigatran therapy is a single level downstream inhibition of thrombin, which could lead to a compensatory increase in upstream clotting factors. Secondly, incomplete inhibition of all available coagulation factors leaves some thrombin activity uninhibited or active. Thirdly, potential drug-drug interactions or inadequate absorption may lead to the unachieved therapeutic levels. Currently, the best strategy of anticoagulation in patients who develop thrombus on dabigatran therapy is uncertain, and a tried therapy with warfarin or left atrial appendage closure should be considered in such patients.

Our report had a few potential limitations. Firstly, our study was underpowered to detect the dabigatran plasma concentration. Secondly, we did not investigate $A B C B 2$ gene polymorphism, which may also impact the blood concentration of dabigatran. Moreover, we did not evaluate P-gp and CES activity in vivo.

\section{CONCLUDING REMARKS}

We suggest that the presence of $A B C B 1$ variant alleles and $C E S$ - 1 variant alleles and drug-drug interaction with atorvastatin

\section{REFERENCES}

Ambudkar, S. V., Kimchi-Sarfaty, C., Sauna, Z. E., and Gottesman, M. M. (2003). P-glycoprotein: from genomics to mechanism. Oncogene 22, 7468-7485. doi: 10.1038/sj.onc.1206948

Antonijevic, N. M., Zivkovic, I. D., Jovanovic, L. M., Matic, D. M., Kocica, M. J., Mrdovic, I. B., et al. (2017). Dabigatran - metabolism, pharmacologic properties and drug interactions. Curr. Drug Metab. 18, 622-635. doi: 10.2174/1389200218666170427113504 are possibly contributing factors for dabigatran therapy failure in this case. Indeed, dabigatran may not suitable for elderly person owing to insufficient renal function and potential drugdrug interaction. Regarding optimal strategy, the treatment with warfarin or left atrial appendage closure may be the viable regimens in such patients suffering dabigatran therapy failure.

More clinical data are required to elucidate the impact of genetic polymorphism on dabigatran pharmacokinetics and thrombosis formation in atrial atrium or left atrial appendage. The impact of $A B C B 1$ and $C E S-1$ gene polymorphism on dabigatran pharmacokinetics should be investigated in a phase 1 clinical study in healthy volunteers based on different races.

\section{AUTHOR CONTRIBUTIONS}

LS was in charge of the treatment of patient. X-YZ was responsible for collecting the patient's information. X-WM was performed the phenotyping test of ABCB1 and CES-1 gene. Z-CG was involved in the care of the patient and interpreted the results, and wrote the manuscript. HL supervised the investigations and interpreted the results, and wrote the manuscript. F-HS was redacted of the manuscript. All authors read and approved the manuscript.

\section{FUNDING}

This work was supported by the Science Fund of Hospital Pharmacy of Shanghai Jiaotong University School of Medicine (JDYX2016ZD003), Program for Key Discipline of Clinical Pharmacy of Shanghai (2016-40044-002), Program for Key but Weak Discipline of Shanghai Municipal Commission of Health and Family Planning (2016ZB0304), and the Fundamental Research Funds for the Central Universities (No.17JCYB11).

\section{ACKNOWLEDGMENTS}

The authors would like to thank the patient for allowing publication of this case.

\section{SUPPLEMENTARY MATERIAL}

The Supplementary Material for this article can be found online at: https://www.frontiersin.org/articles/10.3389/fphar. 2018.00491/full\#supplementary-material 
Crowther, M. A., Julian, J., McCarty, D., Douketis, J., Kovacs, M., Biagoni, L., et al. (2000). Treatment of warfarin-associated coagulopathy with oral vitamin K: a randomised controlled trial. Lancet 356, 1551-1553. doi: 10.1016/S0140-6736(00)03125-1

Dimatteo, C., D’Andrea, G., Vecchione, G., Paoletti, O., Cappucci, F., Tiscia, G. L., et al. (2016). Pharmacogenetics of dabigatran etexilate interindividual variability. Thromb. Res. 144, 1-5. doi: 10.1016/j.thromres.2016.05.025

Favaloro, E. J., Pasalic, L., Curnow, J., and Lippi, G. (2017). Laboratory monitoring or measurement of Direct Oral Anticoagulants (DOACs): advantages, limitations and future challenges. Curr. Drug Metab. 18, 598-608. doi: $10.2174 / 1389200218666170417124035$

Gouin-Thibault, I., Delavenne, X., Blanchard, A., Siguret, V., Salem, J., Narjoz, C., et al. (2016). Inter-individual variability in dabigatran and rivaroxaban exposure: contribution of $\mathrm{ABCB} 1$ genetic polymorphisms and interaction with clarithromycin. J. Thromb. Haemost. 15, 273-283. doi: 10.1111/jth.13577

Johnson, J. A., Gong, L., Whirl-Carrillo, M., Gage, B. F., Scott, S. A., Stein, C. M., et al. (2011). Clinical pharmacogenetics implementation consortium guidelines forCYP2C9 and VKORC1 genotypes and warfarindosing. Clin. Pharmacol. Ther. 90, 625-629. doi: 10.1038/clpt.2011.185

Kishimoto, W., Ishiguro, N., Ludwig-Schwellinger, E., Ebner, T., and Schaefer, O. (2014). In vitro predictability of drug-drug interaction likelihood of P-glycoprotein-mediated efflux of dabigatran etexilate based on [I]2/IC50 threshold. Drug Metab. Dispos. Biol. Fate Chem. 42, 257-263. doi: 10.1124/dmd.113.053769

Laizure, S. C., Parker, R. B., Herring, V. L., and Hu, Z. Y. (2014). Identification of carboxylesterase-dependent dabigatran etexilate hydrolysis. Drug Metab. Dispos. Biol. Fate Chem. 42, 201-206. doi: 10.1124/dmd.113. 054353

Liesenfeld, K. H., Lehr, T., Dansirikul, C., Reilly, P. A., Connolly, S. J., Ezekowitz, M. D., et al. (2011). Population pharmacokinetic analysis of the oral thrombin inhibitor dabigatran etexilate in patients with non-valvular atrial fibrillation from the RE-LY trial. J. Thromb. Haemost. 9, 2168-2175. doi: 10.1111/j.1538-7836.2011.04498.x

López-López, J. A., Sterne, J. A. C., Thom, H. H. Z., Higgins, J. P. T., Hingorani, A. D., Okoli, G. N., et al. (2017). Oral anticoagulants for prevention of stroke in atrial fibrillation: systematic review, network meta-analysis, and cost effectiveness analysis. BMJ 359:j5058. doi: 10.1136/bmj.j5058

Luis, S. A., Poon, K., Luis, C., Shukla, A., Bett, N., and Hamilton-Craig, C. (2013). Massive left atrial thrombus in a patient with rheumatic mitral stenosis and atrial fibrillation while anticoagulated with dabigatran. Circ. Cardiovasc. Imaging 6, 491-492. doi: 10.1161/CIRCIMAGING.113. 000253

Mega, J. L., and Simon, T. (2015). Pharmacology of antithrombotic drugs: an assessment of oral antiplatelet and anticoagulant treatments. Lancet 386, 281-291. doi: 10.1016/S0140-6736(15)60243-4

Paré, G., Eriksson, N., Lehr, T., Connolly, S., Eikelboom, J., Ezekowitz, M. D., et al. (2013). Genetic determinants of dabigatran plasma levels and their relation to bleeding. Circulation 127, 1404-1412. doi: 10.1161/CIRCULATIONAHA.112.001233
Pirmohamed, M., Burnside, G., Eriksson, N., Jorgensen, A. L., Toh, C. H., Nicholson, T., et al. (2013). A randomized trial of genotype-guided dosing of warfarin. N. Engl. J. Med. 369, 2294-2303. doi: 10.1056/NEJMoa1311386

Redinbo, M. R., Bencharit, S., and Potter, P. M. (2003). Human carboxylesterase 1: from drug metabolism to drug discovery. Biochem. Soc. Trans. 31, 620-624. doi: 10.1042/bst0310620

Ross, S., and Paré, G. (2013). Pharmacogenetics of antiplatelets and anticoagulants: a report on clopidogrel, warfarin and dabigatran. Pharmacogenomics 14, 1565-1572. doi: 10.2217/pgs.13.149

Shah, P., Mithawala, P., Konlian, D., Soyombo, A., and Bikkina, M. (2015). Thrombus formation in left atrium on dabigatran therapy. Case Rep. Cardiol. 2015:518982. doi: 10.1155/2015/518982

Sharma, S., Singh, S., Sandhu, R., Monterroso, M., Bhambi, N., and Sharma, R. (2014). Case report series of left atrial thrombus formation in patients on dabigatran therapy. Am. J. Ther. 21, e71-e74. doi: 10.1097/MJT.0b013e3182491e14

Stangier, J., and Clemens, A. (2009). Pharmacology, Pharmacokinetics, and Pharmacodynamics of Dabigatran Etexilate, an Oral Direct Thrombin Inhibitor. Clin. Appl. Thromb. Hemost. 15 (Suppl. 1), 9S-16S. doi: $10.1177 / 1076029609343004$

Stöllberger, C., and Finsterer, J. (2015). Relevance of P-glycoprotein in stroke prevention with dabigatran, rivaroxaban, and apixaban. Herz 40, 140-145. doi: 10.1007/s00059-014-4188-9

Verhalen, B., Dastvan, R., Thangapandian, S., Peskova, Y., Koteiche, H. A., Nakamoto, R. K., et al. (2017). Energy transduction and alternating access of the mammalian ABC transporter P-glycoprotein. Nature 543, 738-741. doi: $10.1038 /$ nature21414

Wadkins, R. M., Morton, C. L., Weeks, J. K., Oliver, L., Wierdl, M., Danks, M. K., et al. (2001). Structural constraints affect the metabolism of 7-ethyl10-[4-(1-piperidino)-1-piperidino]carbonyloxycamptothecin (CPT-11) by carboxylesterases. Mol. Pharmacol. 60, 355-362. doi: 10.1124/mol.60.2.355

Wessler, J. D., Grip, L. T., Mendell, J., and Giugliano, R. P. (2013). The Pglycoprotein transport system and cardiovascular drugs. J. Am. Coll. Cardiol. 61, 2495-2502. doi: 10.1016/j.jacc.2013.02.058

Wysowski, D. K., Nourjah, P., and Swartz, L. (2007). Bleeding complications with warfarin use: a prevalent adverse effect resulting in regulatory action. Arch. Intern. Med. 167, 1414-1419. doi: 10.1001/archinte.167.13.1414

Conflict of Interest Statement: The authors declare that the research was conducted in the absence of any commercial or financial relationships that could be construed as a potential conflict of interest.

Copyright (c) $2018 \mathrm{Gu}, \mathrm{Ma}$, Zheng, Shen, Shi and Li. This is an open-access article distributed under the terms of the Creative Commons Attribution License (CC $B Y)$. The use, distribution or reproduction in other forums is permitted, provided the original author(s) and the copyright owner are credited and that the original publication in this journal is cited, in accordance with accepted academic practice. No use, distribution or reproduction is permitted which does not comply with these terms. 